\title{
Sistema de alerta temprana por movimiento en masa inducido por lluvia para Ciudad Bolívar (Colombia)
}

\author{
Early Warning System for Rain-Induced Mass \\ Movement for Ciudad Bolívar (Colombia)
}

David Esneider Gutiérrez Alvis ${ }^{1}$, Leydy Stefany Bornachera Zarate ${ }^{2}$, Darin Jairo Mosquera Palacios ${ }^{3} \bowtie$

\footnotetext{
${ }^{1}$ Universidad Distrital Francisco José de Caldas, Bogotá, Colombia

2 Universidad Distrital Francisco José de Caldas, Bogotá, Colombia

${ }^{3}$ Universidad Distrital Francisco José de Caldas, Bogotá, Colombia
}

Grupo de investigación ORION, Calle 68D Bis A Sur n. ${ }^{\circ}$ 49F-70, Facultad Tecnológica, Universidad Distrital Francisco José de Caldas, Bogotá, Colombia. Email: djmosquerap@uditrital.edu.co

Recibido: mayo 21 del $2018 \quad$ Aprobado: agosto 8 del $2018 \quad$ Disponible en línea: septiembre 1 del 2018

Cómo citar este artículo: D. E. Gutiérrez-Alvis, L. Bornachera-Zarate y D. J. Mosquera-Palacios, "Sistema de alerta temprana por movimiento en masa inducido por Iluvia para Ciudad Bolívar (Colombia)", Revista Ingeniería Solidaria, vol. 14, no. 26, 2018. doi: https://doi.org/10.16925/in.v14i26.2453

\section{Resumen}

Introducción: este es un artículo de investigación en el que se crea un sistema de alerta temprana por movimiento en masa inducido por lluvia para la localidad de Ciudad Bolívar, en Bogotá, Colombia, desarrollado en la Universidad Distrital Francisco José de Caldas en el año 2018.

Metodología: se desarrolló una red de sensores por medio de una estación base, que se encarga de generar una alerta si existe riesgo de deslizamiento.

Resultados: Ios datos generados por la red de sensores, como humedad y temperatura, generan alertas aumentando la posibilidad de salvar vidas de personas vulnerables en la localidad.

Conclusión: con este proyecto, se busca salvar lo más importante del ser humano: la vida; además de concientizar a la gente del peligro que conlleva construir casas en lugares con alto riesgo de deslizamiento, se pretende mostrar que por medio de la tecnología es posible salvar vidas, alertando con tiempo catástrofes naturales como los deslizamientos.

Originalidad: en este trabajo, se genera un estudio de suelo previo en la localidad de Ciudad Bolívar y se crea una escala de alerta propia para el sistema, implementando en la localidad el Sistema de Información para la Gestión del Riesgo y Cambio Climático (SIRE).

Limitaciones: el escaso desarrollo de los sistemas de alerta en deslizamiento de tierra inducido por Iluvia en Colombia.

Palabras clave: desastres naturales, deslizamiento de tierra, escala de alerta, estación base, movimiento en masa, res de sensores. 


\title{
Early Warning System for Rain-Induced Mass Movement for Ciudad Bolívar (Colombia)
}

\author{
Abstract \\ Introduction: This research article introduces an early warning system for rain-induced mass mo- \\ vement for the district of Ciudad Bolívar, in Bogotá, Colombia, developed at the Universidad Distrital \\ Francisco José de Caldas in 2018. \\ Methods: A sensor network was developed by means of a base station, which is responsible for issuing \\ a warning if there is a risk of landslide. \\ Results: The data provided by the sensor network, such as humidity and temperature, issue warnings \\ to increase the possibility of saving the lives of vulnerable population in the district. \\ Conclusion: This project seeks to save the most important thing a human being has: his life. In addition \\ to raising awareness among individuals of the danger of building houses in places with a high risk \\ of landslides, the aim is to show that through technology we could save lives, warning about natural \\ disasters such as landslides. \\ Originality: In this work, a previous soil survey was conducted in Ciudad Bolívar and a warning scale \\ was created for the system, implementing the Risk Management and Climate Change Information \\ System (SIRE) in the district. \\ Limitations: Scarce development of rain-induced landslide warning systems in Colombia. \\ Keywords: natural disasters, landslide, warning scale, base station, mass movement, sensor network.

\section{Sistema de alerta precoce para movimentos de massa induzidos pela chuva para Ciudad Bolívar (Colômbia)}

\section{Resumo}

Introdução: neste artigo de pesquisa, cria-se um sistema de alerta precoce para os movimentos de massa induzidos pela chuva para Ciudad Bolívar, uma área em Bogotá, Colômbia. Foi desenvolvido na Universidad Distrital Francisco José de Caldas em 2018.

Metodologia: uma rede de sensores foi desenvolvida por meio de uma estação base, responsável por gerar um alerta em caso de risco de deslizamento de terra.

Resultados: os dados gerados pela rede de sensores, como umidade e temperatura, produzem alertas que ajudam a aumentar a possibilidade de salvar vidas de pessoas vulneráveis na área.

Conclusão: este projeto procura salvar o aspecto mais importante para os seres humanos: a vida. Além disso, visa conscientizar as pessoas sobre o perigo de construir casas em locais com alto risco de deslizamento de terra. 0 objetivo é mostrar que a tecnologia pode ajudar a salvar vidas graças aos alertas precoces de desastres naturais, como deslizamentos de terra.

Originalidade: este trabalho desenvolve um estudo prévio do solo na área de Ciudad Bolívar e, em seguida, gera uma escala de alerta para o sistema, implementando assim o Sistema de Informação para Gestão de Riscos e Mudança do Clima (SIRE).

Limitações: 0 escasso desenvolvimento de sistemas de alerta de deslizamentos de terra causados pela chuva na Colômbia.

Palavras-chave: desastres naturais, deslizamento de terra, escala de alerta, estação base, movimento de massa, rede de sensores. 


\section{Introducción}

Constantemente, se observan cambios no solo en el diario vivir, sino también en el medio ambiente, sobre todo en el clima. Muestra de ello es el invierno, estación en que los días son más fríos y es la época de mayor frecuencia de lluvia [1]. Esta situación causa miles de muertes anualmente. Por ejemplo, en el estado Vargas, en Venezuela, las lluvias han causado un aproximado de 10000 víctimas, según cifras oficiales, y un enorme costo en pérdidas de infraestructura. Encendemos la televisión o leemos los periódicos y son más frecuentes las noticias alusivas a desastres naturales, terremotos, tsunamis, vendavales e inundaciones que cobran la vida de muchas personas en todo el mundo.

Otro ejemplo de ello, de acuerdo con GálvezHooper [2], son las inundaciones y los deslizamientos en la parte alta de la cuenca del río Chiriquí Viejo, en Panamá: pocas personas se detienen a revisar el porqué de estos cambios y otras solamente lo ignoran, pero todos conocemos las causas y soluciones a estas crisis que cada día van en aumento, sin hacer nada al respecto.

Cuando la tecnología avanza, se abren nuevas esperanzas, como el uso de las herramientas del Sistema de Información Geográfica (sIG) [3], que muestra la capacidad de la tecnología de ser aliada en la predicción y la prevención de las amenazas a los desastres por eventos extremos de fácil uso y manejo, con el fin de detectar zonas vulnerables o propensas a derrumbes. Una de esas esperanzas se llama tecnología ambiental (red de sensores). Castro et al. [4] analizan los fenómenos naturales peligrosos, previniendo sus efectos en las actividades de los habitantes, sus bienes y en la infraestructura existente.

En la ciudad de Bogotá, existen localidades como Suba, Usaquén, Chapinero, Santa Fe, San Cristóbal, Usme y Ciudad Bolívar, con riesgo inminente de deslizamientos de tierra, que aún no cuentan con un monitoreo ni con un Modelo Sistémico a Deslizamiento Superficial de Suelos [5], y cada año el invierno deja decenas de víctimas mortales.

De tal modo, el foco de trabajo será la localidad más vulnerable: Ciudad Bolívar. En este proyecto, se pretende encontrar una solución tecnológica con las redes de sensores por medio de una estación base que con mensajes de alerta busca informar con antelación a las entidades especializadas en estos eventos cuando los parámetros de las escalas sobrepasen los porcentajes estándar, según lo registra Pradenas [6], mediante la determinación de umbrales críticos que relacionan las precipitaciones con los deslizamientos; esto con el fin de que se tomen las medidas necesarias para salvar el mayor número de vidas.

\subsection{Antecedentes de investigación}

Bravo et al. [1], en su investigación, desarrollaron un sistema de alerta temprana en el estado Vargas, en Venezuela, con la instalación de 65 pluviómetros ubicados a lo largo de las comunidades que recogen datos de lluvia. Estos son cargados a través de un portal de la página web del Instituto Nacional de Meteorología e Hidrología (INAMEH). Así mismo, se realizó la implementación de limnímetros ubicados en lechos de ríos y quebradas, también en paredes y postes de luz donde han quedado las marcas de inundaciones pasadas.

Gálvez-Hooper [2] evaluó el riesgo de inundaciones y deslizamientos en la zona de estudio para generar información de base que cubra aspectos de identificación de áreas susceptibles a riesgos por deslizamiento (metodología de Vahrson Mora) e inundaciones (modelo de simulación Floodarea y mapeo comunitario), así como información sobre las percepciones locales y las estrategias para reducir los riesgos. Se caracterizaron los eventos históricos, se estimó la vulnerabilidad y se ubicaron las áreas más vulnerables cerca de la cuenca alta del río Chiriquí Viejo, provincia de Chiriquí, Panamá.

Yábar [3] promueve la investigación y proporciona una base de pruebas a la comunidad internacional sobre la carga de morbilidad y problemas de salud relacionados con desastres y conflictos, a fin de mejorar la preparación y las respuestas a estas emergencias humanitarias. El CRED capacita a gerentes de campo, estudiantes, personal de socorro y profesionales de la salud en la gestión de emergencias humanitarias a corto y a largo plazo.

Así mismo, Yábar [3] creó una metodología para la planificación de un Sistema de Alerta Temprana (SAT) de inundaciones para la región de Madre de Dios, en Perú, con uso de las herramientas SIG, mostrando la capacidad de la tecnología de ser aliada en la predicción y prevención de amenazas de desastres por eventos extremos de fácil uso y manejo, con el fin de detectar zonas vulnerables o propensas a inundaciones. Las herramientas usadas son ArcGis 10 con las extensiones 
3D Analyst y HEC-Georas, así como el software de modelación hidrológica HEC-RAs.

Castro et al. [4] investigaron los peligros naturales en geositios de interés patrimonial en la la Costa Sur del desierto marginal de Atacama, entre Chañaral y el río Copiapó, en Chile. Los riesgos naturales presentaron una gran diversidad de formas, tipos de rocas y yacimientos fosilíferos, entre los que destacan algunos geositios, lugares significativos para la geociencia, la cultura y el turismo de naturaleza. Los geositios constituyen un recurso de importancia patrimonial con potencial para la conservación y el soporte de actividades turísticas sustentables en la región de Atacama. La vulnerabilidad del medio morfo dinámico árido de la zona costera se manifiesta en la ocurrencia de peligros naturales por sismicidad y tsunamis, remoción en masa y flujos. Se caracterizan y analizan los fenómenos naturales peligrosos de la costa sur de Atacama previniendo sus efectos tanto en las actividades de los habitantes, en sus bienes y en la infraestructura existente.

Pradena [6] desarrolló un umbral empírico de deslizamiento por precipitación para la provincia de Concepción, en Chile. Los deslizamientos de terreno son procesos frecuentes en Chile y en el mundo, provocados por factores como procesos geológicos y geomorfológicos, y por estímulos externos como la lluvia. La posibilidad de ocurrencia de un deslizamiento comienza desde el momento cuando se forma una ladera natural o se construye un talud artificial. Lo anterior ha conducido a numerosos estudios en el mundo sobre el tema de las amenazas por deslizamiento y la estabilidad de taludes, mediante la determinación de umbrales críticos que relacionan las precipitaciones con los deslizamientos.

Peña-Rincón [5] mostró un monitoreo y un Modelo Sistémico a Deslizamiento Superficial de Suelos para Manizales, en Colombia, creando una propuesta para el monitoreo de deslizamiento de suelos y un estudio desde la dinámica de sistemas para integrar los deslizamientos superficiales y profundos.

Cardozo [7] creó una zonación de susceptibilidad por procesos de remoción en masa en la cuenca del río Tartagal, presentando el primer mapa de zonación de susceptibilidad por procesos de remoción en masa para la cuenca del río Tartagal (Salta, Argentina), usando técnicas de sensores remotos y un SIG. La superficie afectada y el volumen del material removido durante el alud del 2009 causaron decenas de muertes en esta comunidad.

Proaño-Pachucho [8] analizó los componentes estructurales y funcionales para el diseño de un sistema de alerta integral a nivel institucional por potenciales inundaciones en el Distrito Metropolitano de Quito, en Ecuador, el cual presenta precipitaciones en periodos muy cortos de tiempo, sobre todo en los meses de marzo, abril, mayo y octubre. Este fenómeno haría que las capacidades de conducción de agua lluvia del alcantarillado, y de drenajes y absorción de los suelos sean sobrepasadas y que se generen inundaciones en las partes bajas, planas y quebradas de la ciudad. La vulnerabilidad aumenta o disminuye en la medida en que la sociedad, las instituciones u organizaciones de respuesta están lo suficientemente preparadas para minimizar la exposición a una amenaza ante inundaciones. Para esto, se han construido obras de infraestructura, regulación hídrica y retención de sólidos en las laderas y quebradas que permiten prevenir y mitigar los riesgos.

Carbajal [9] dio a conocer el riesgo de deslizamientos ocasionados por las precipitaciones en la microcuenca de Comas, en Concepción, Chile, ubicada en el departamento de Junín, en dos distritos: Comas, provincia de Concepción, e Ingenio, provincia de Huancayo, entre altitudes de 3060 msnm y 4800 msnm. Dadas sus características geográficas, geológicas, geomorfológicas, climáticas y socioeconómicas, es altamente vulnerable a los deslizamientos porque periódicamente recibe intensas precipitaciones que conducen a un riesgo de deslizamientos, ocasionando serios problemas agrícolas y sociales. Entonces, el manejo de las cuencas y microcuencas debe tener como enfoque fundamental la reducción de este riesgo, principalmente la asociada a fenómenos hidroclimáticos, inundaciones, deslizamientos, avalanchas y sequías, entre otros.

\section{Contextualización}

En Colombia, hay varios sectores donde no se es permitido habitar debido al riesgo inminente de derrumbes. A causa del desplazamiento forzado por grupos armados ilegales, muchas personas han migrado a Bogotá [10] y, según los datos existentes, el 93\% de la población desplazada en Colombia lo 
ha hecho hacia áreas urbanas. Al mismo tiempo, durante la última década, el país ha experimentado un intenso proceso de migración desde zonas rurales. Bogotá cuenta con unos siete millones de habitantes, incluyendo el mayor número de inmigrantes y desplazados internos del país (alberga a 270000 desplazados internos).

A su vez, quienes llegan a la capital construyen en sectores de mucho riesgo, cuyos suelos muy inestables, y donde, sumándole lluvias, hay una alta probabilidad de deslizamientos. Además, hay una gran cantidad de población construyendo en lugares reducidos, con materiales artesanales en su mayoría y sin la respectiva planeación autorizada por el Distrito, esto propicia que el riesgo aumente, sobre todo en épocas de invierno.

El presente trabajo de investigación tiene como objetivo crear un sistema de alerta temprana por movimiento en masa inducido por lluvia para Ciudad Bolívar, que alerte e informe cuando haya una alerta de deslizamiento de tierra por lluvias en la localidad. Se toma este sector de la ciudad por su ubicación geográfica y por el riesgo inminente a causa de la sobrepoblación. Este proyecto se realizará por medio de una red de sensores instalada en la zona de posibles emergencias. El principal reto es captar e interpretar a tiempo toda la información suministrada por la red de sensores para así tomar decisiones rápidas.

El método utilizado consiste en el diseño de una red de sensores que mida la humedad del suelo, teniendo en cuenta los estudios de suelo previos, y además identificar cuántos metros cúbicos de agua soporta el suelo, hasta el punto de deslizamiento. Con estos datos, se pretende calcular un rango de mediciones que nos genere alertas tempranas a fin de tener un lapso pertinente para informar a la comunidad el desalojo inmediato de sus viviendas. Toda esta información será posible interpretarla por medio de sistemas de información creados para este proyecto.

Este trabajo se llevó a cabo en la localidad de Ciudad Bolívar en Bogotá, la cual cuenta con una extensión total de 12999 ha de las cuales 3391 ha se clasifican como suelo urbano y 9608 ha corresponden al suelo rural, que equivale al $73,9 \%$ del total de la superficie de la localidad. Después de Sumapaz y Usme, Ciudad Bolívar está clasificada como la localidad más extensa, está ubicada en una altitud de $2400 \mathrm{msnm}$ en la parte más baja y de $3100 \mathrm{msnm}$ en la parte más alta, con temperatura mínima de $9^{\circ} \mathrm{C}$ y máxima de $14^{\circ} \mathrm{C}$.

Por localidades, la mayor concentración de víctimas tiene lugar en Ciudad Bolívar (28 813), seguida de Bosa (21 067), Kennedy (20 659), Suba (15 251), San Cristóbal (13 003), Usme (12 112), Engativá (8387), Rafael Uribe Uribe (8199), Usaquén (4894), Tunjuelito (3997), Fontibón (3671), Los Mártires (3588) y Santa Fe (2828) [11].

Con base en tan alto número de zonas con riesgo de deslizamiento de tierra, se construye la estación base a fin de recopilar toda la información que ayude a disminuir el número de muertes [12], ya que solo en Cundinamarca tenemos los datos de la tabla 1.

A partir de un buen uso de la tecnología, se busca reducir estas cifras. Se sabe que si no se hace nada respecto a la problemática del calentamiento global, los deslizamientos aumentarán y por ende las víctimas mortales.

\section{Características físicas}

- Temperatura: 14 grados promedio anual

- Humedad relativa: seca y semiseca

- Precipitación total: 600 a $800 \mathrm{~mm}$ anuales

- Sector contra los cerros del sur de la ciudad: 800 a $1.000 \mathrm{~mm}$ anuales

- Altimetría: entre 2650 y 3100 msnm

Tabla 1. Indicadores de catástrofes de Cundinamarca, Antioquia y Caldas

\begin{tabular}{cccccc}
\hline & Departamento & Población & Eventos & Muertos & Afectados \\
\hline 1 & Antioquia & 6456299 & 1384 & 586 & 490459 \\
2 & Cundinamarca & 2680041 & 2206 & 243 & 264539 \\
3 & Caldas & 987991 & 507 & 223 & 130914 \\
\hline
\end{tabular}

Fuente: elaboración propia 


\section{Temperatura media de Ciudad Bolivar en el 2017}

La estación climatológica Simón Bolívar, que cubre esta localidad, permite establecer la temperatura en un promedio de $14,1^{\circ} \mathrm{C}$, con un mínimo de $9^{\circ} \mathrm{C}$ y un máximo de $19,4^{\circ} \mathrm{C}$.

\section{Temperatura ${ }^{\circ} \mathrm{C}$}

En la mayor parte del territorio de Bogotá, se registra un nivel de precipitación anual de 600 a 800 mm; sin embargo, en Ciudad Bolívar se registran precipitaciones entre 800 y $1.000 \mathrm{~mm} / \mathrm{año}$, siendo la localidad de Bogotá donde más llueve. Los meses en los que más llueve son abril, mayo, octubre, noviembre y diciembre, cambios debidos al calentamiento global.

\section{Precipitaciones $(\mathrm{mm})$}

Dependiendo de la relación precipitación-brillo solar, se presenta la condición de zona húmeda en la parte suroriental de la localidad, que abarca los cerros de la ciudad, y una porción de territorio con condiciones de zona seca en la parte noroccidental, a lo largo del límite con las localidades de Tunjuelito y Bosa.

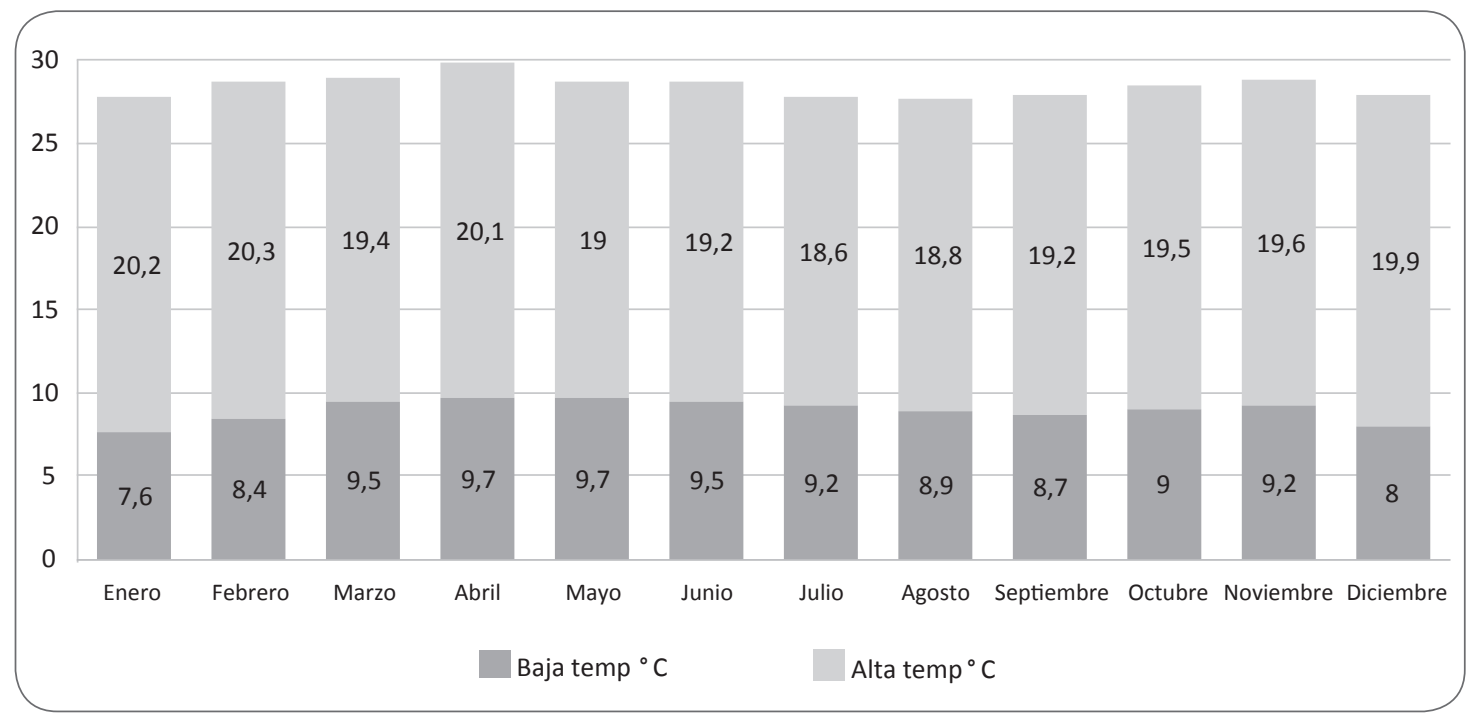

Figura 1. Temperatura media de la localidad de Ciudad Bolívar 2017

Fuente: elaboración propia

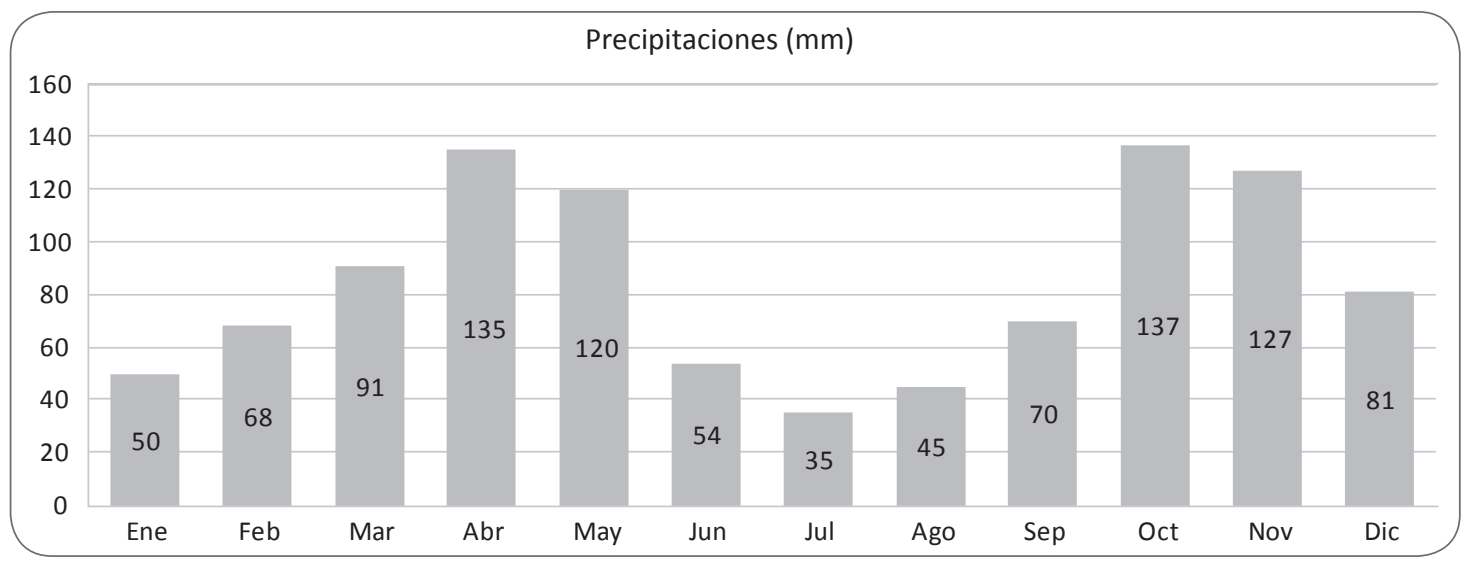

Figura 2. Precipitación media de la localidad de Ciudad Bolívar 2017

Fuente: elaboración propia 
El promedio de humedad de Ciudad Bolívar en el 2017 fue de 75,5\%, y los meses cuando hubo el mayor número de días lluviosos fue en invierno, del miércoles 21 de diciembre del 2016 al lunes 20 de marzo del 2017 según lo indicado por el Instituto de Hidrología, Meteorología y Estudios Ambientales (Ideam).

\section{Humedad (\%)}

Con base en esta información, los meses con mayor riesgo de deslizamientos son aquellos entre finales de diciembre a finales de marzo.

\section{Hidrología}

En Ciudad Bolívar, según Hermelin [13], hay un alto número de quebradas sin canalizar, las cuales se convierten en focos de enfermedades para la población de la localidad. Se destaca la Laguna Terreros, ubicada hacia el occidente de la localidad en la zona montañosa.

El principal recurso hídrico de la localidad es la cuenca del río Tunjuelo, la cual recibe afluentes como las quebradas Limas, Trompeta, La Estrella y El Infierno. A pesar de que la cuenca es aprovechada en su parte alta para el acueducto de Bogotá, al pasar por la ciudad, se convierte en un receptor de aguas lluvias y negras fuertemente contaminadas, ocasionado por la no existencia de redes troncales de drenaje, con lo que la evacuación se hace directamente al río Tunjuelito a través de calles, zanjas y vallados.

Para medir la escala de riesgo en Ciudad Bolívar, fue necesario remitirse a la plataforma del Sistema de Información para la Gestión del Riesgo y Cambio Climático (SIRE). Aquí se encuentra la escala de lluvias en la localidad y la precipitación por milímetros de agua. Con base en esta escala, se puede determinar cuándo hay alerta por lluvia.

Los sensores son dispositivos hardware que producen una respuesta medible ante un cambio físico, como temperatura, presión y humedad. Los sensores detectan o miden cambios físicos en el área que están monitorizando. La señal analógica continúa detectada, es digitalizada por un convertidor analógico digital y es enviada a un controlador para ser procesada.

Las características y los requerimientos que un sensor debe tener son: tamaño pequeño, un consumo bajo de energía, operar en densidades volumétricas altas, ser autónomo y tener capacidad para adaptarse al ambiente. Los sensores pueden estar clasificados en tres categorías:

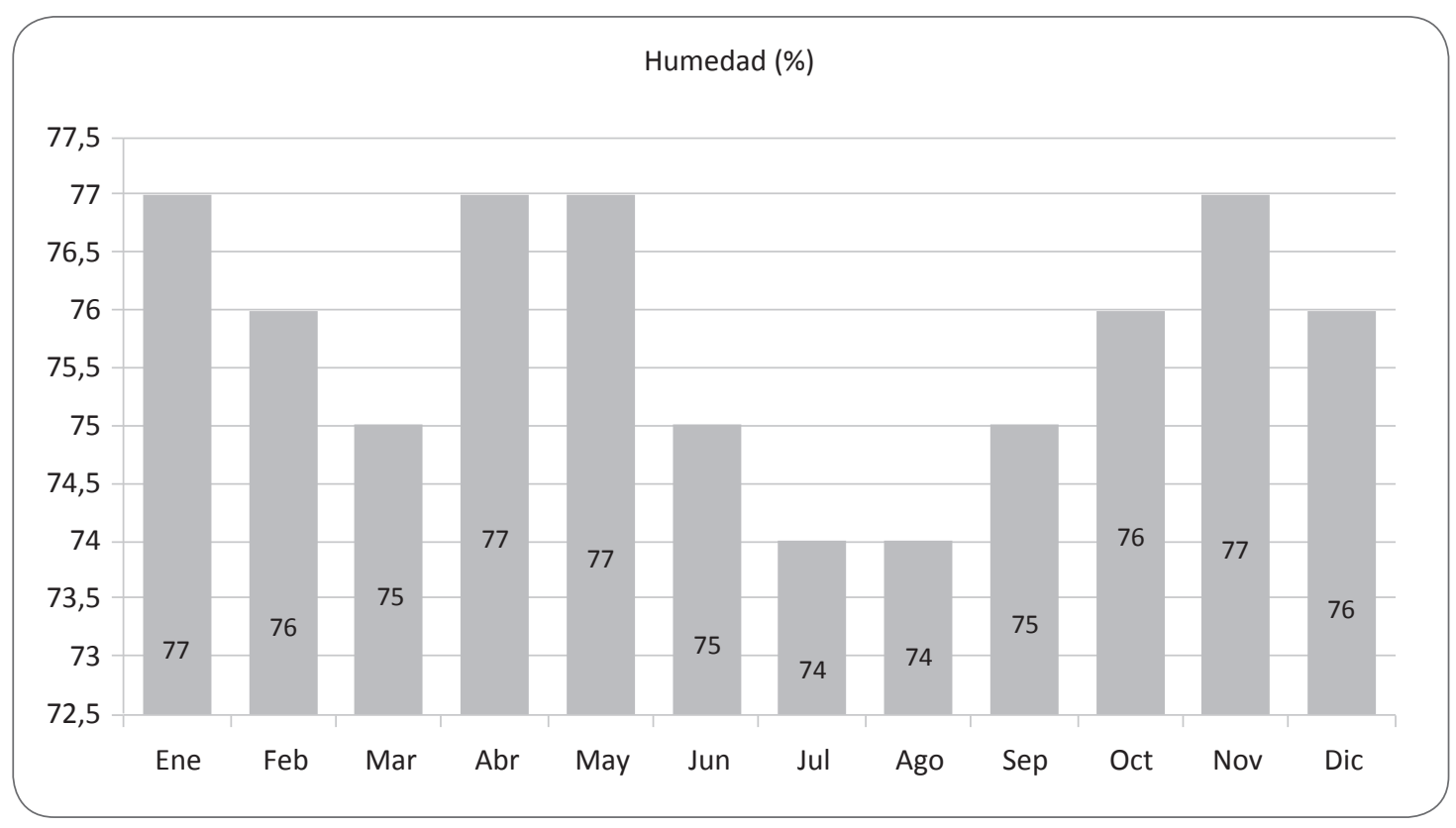

Figura 3. Humedad media de la localidad de Ciudad Bolívar 2017

Fuente: elaboración propia 
Sensores pasivos omnidireccionales: captan los datos sin necesidad de manipular el entorno. Son autoalimentados y solo usan la energía para amplificar la señal analógica captada. No hay ninguna noción de 'dirección' involucrada en estas mediciones.

Sensores pasivos unidireccionales: tienen bien definida la dirección desde donde deben captar la información. Un ejemplo típico es una cámara.

Sensores activos: sondean el ambiente, por ejemplo, un radar o un sonar o algún tipo de sensor sísmico que genera ondas expansivas a través de pequeñas explosiones.

En el proyecto, se utilizaron sensores activos ya que brindan información las veinticuatro horas del día, en tiempo real y alertando en caso de emergencia.

Los nodos (WSN) de red de sensores están típicamente organizados en uno de tres tipos de topologías de red. La utilizada en el proyecto es la topología tipo malla, cuya característica es que los nodos se pueden conectar a múltiples nodos en el sistema y pasar los datos por el camino disponible de mayor confiabilidad.

Para este proyecto, se decidió trabajar con topología de malla, ya que brinda mayor confiabilidad y en dado caso la información que se recolecta no tendría pérdida, pues si algún nodo se daña o la información se pierde, el proceso no queda estancado, dado que el nodo de al lado tendría la misma información y así llegaría con éxito a la estación base.

Los sensores utilizados en el proyecto fueron sensor de humedad FC28 y sensor de temperatura LM35, ubicando todos los sensores en malla para que todos los datos lleguen a la estación base sin perderse en el camino.

¿Qué pasa si un sensor se daña? Como la topología de red utilizada es la red en malla, en la cual todos los sensores van conectados a la estación base y todos conocen la información del otro, en caso de falla, el sensor más cercano tiene la misma información del sensor dañado.

\section{Metodología}

Para el desarrollo del proyecto, se creó una estación base capaz de medir la humedad del suelo y la temperatura ambiente, para ello se utilizaron:

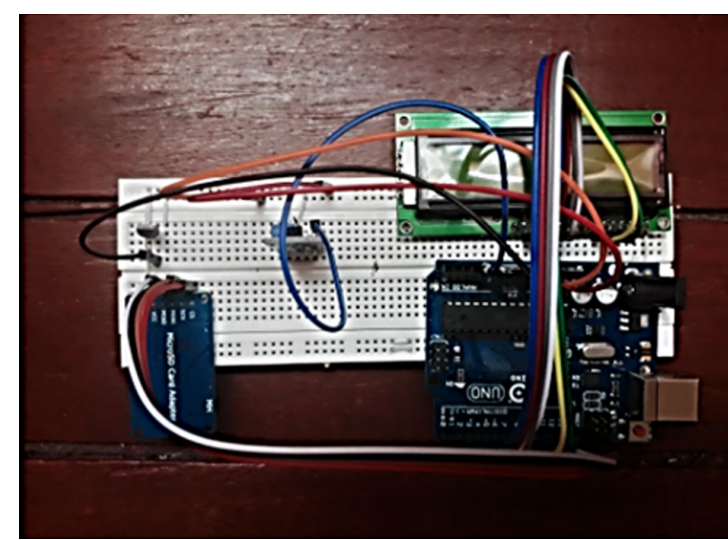

Figura 4. Estación base medidor de humedad y temperatura Fuente: elaboración propia

Para determinar la escala de alerta de humedad, fue necesario generar ciertas conclusiones, ya que no hay información que produzca esta escala, debido a la variedad del suelo que depende de la zona a trabajar; por ejemplo, si el lugar de monitoreo está cerca de cuencas, la humedad va a variar.

Por ello, se tomaron como base los deslizamientos que ocurrieron en la localidad de Ciudad Bolívar el 28 de marzo de 2017 en el barrio J.J. Rendón, en donde se obtuvieron los siguientes datos acerca del clima: precipitación de $135 \mathrm{~mm}$ de agua, humedad de $80 \%$, temperatura promedio de $14,3{ }^{\circ} \mathrm{C}$.

- Humedad igual o mayor a $80 \%=$ Alerta

- Precipitación de más de $130 \mathrm{~mm}$ de agua $=$ Alerta

Con base en esta información, ponemos como escala de alerta mínima los anteriores valores para el proyecto.

\section{Diagrama de flujo}

Para el funcionamiento de la estación base, fue necesaria la programación del Arduino, que se mostrará en el diagrama de flujo de la figura 5. Aquí se muestra el inicio del proceso y después de declarar las librerías, se declaran las variables de temperatura y humedad. En los recuadros azules, se asignan los pines que cada una de las variables utilizará en el Arduino, además de asignar los minutos de espacio que mostrarán los datos. 
Se evidencian dos opciones: que la tarjeta SD que está en la estación base no inicializa o que inicializa exitosamente.

En caso de no inicializar, mostrará error y es necesario volverlo a compilar. Si es el caso contrario e inicia exitosamente, de manera automática genera el archivo datos.txt con los datos obtenidos.

En el cuadro de la mitad, se pregunta si generó archivo. Si lo generó, muestra un mensaje que dice "Escribiendo SD", en el que mostrará la temperatura, la humedad y su valor en tiempo real. En caso de no generar archivo, es necesario repetir el proceso.

En el diagrama de flujo, encontramos las instrucciones dadas en forma de código para que el Arduino lo pueda procesar.

\section{Código de montaje}

Se declaran las librerías para utilizar cada dispositivo utilizado en el montaje y el respectivo código declarando las variables.

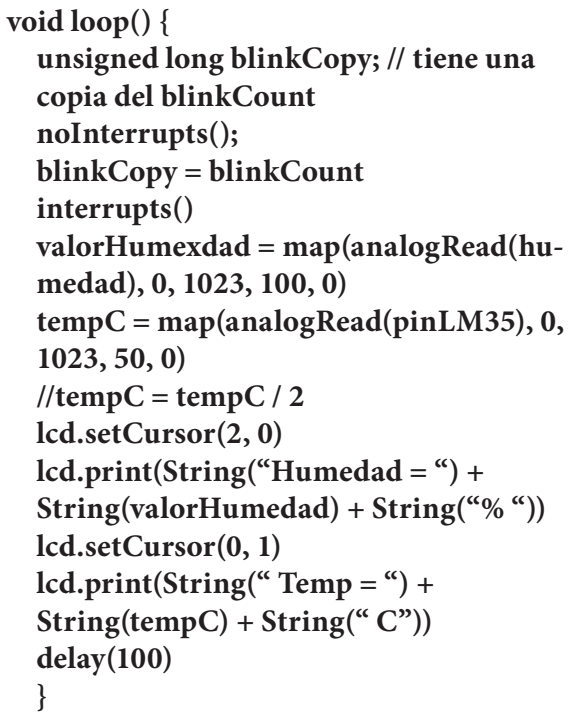

Figura 6. Función principal del código del proyecto sistema de alerta temprana por movimiento en masa inducido por lluvia para la localidad de Ciudad Bolívar Fuente: elaboración propia

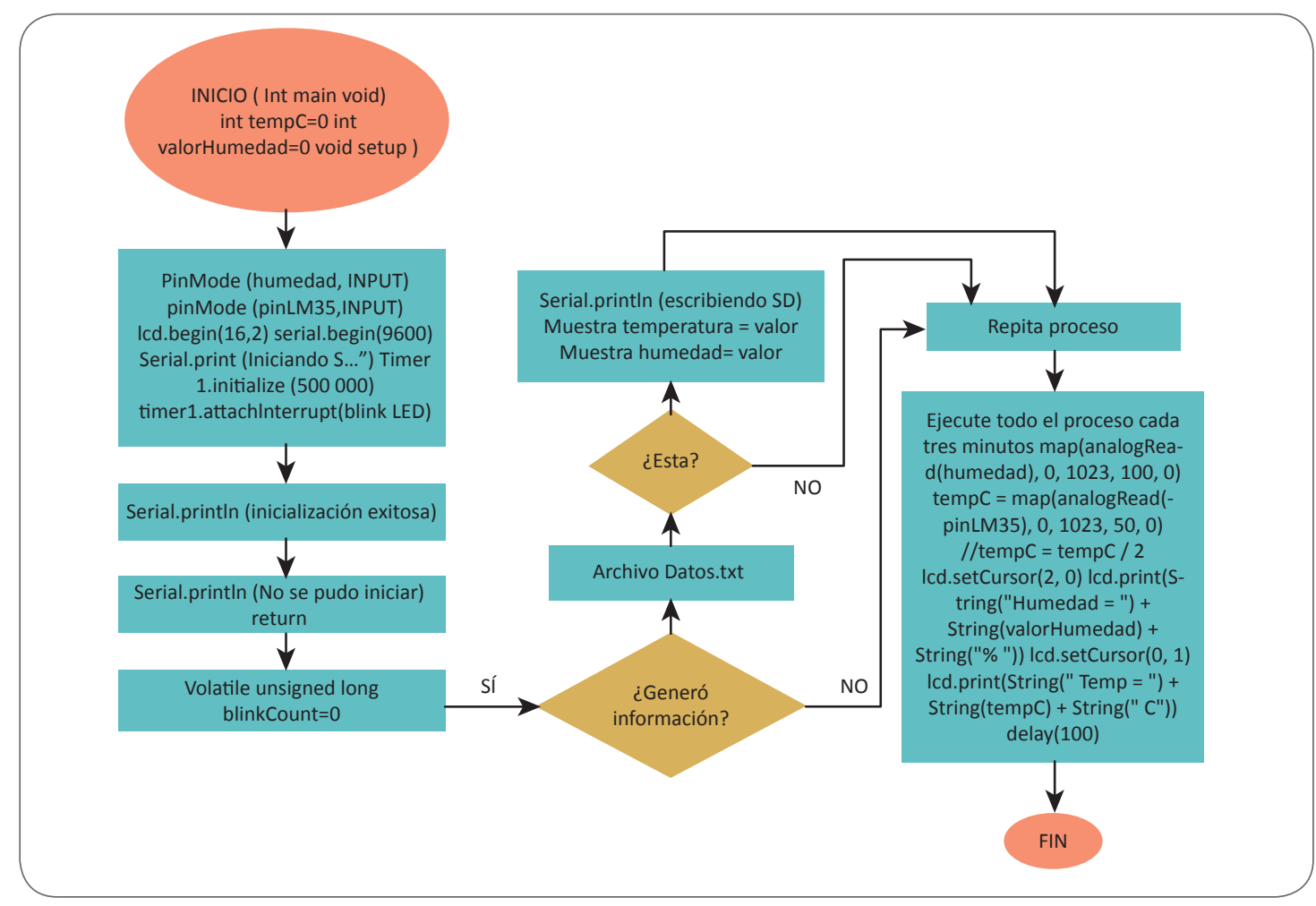

Figura 5. Diagrama de flujo del proyecto sistema de alerta temprana por movimiento en masa inducido por lluvia para la localidad de Ciudad Bolívar

Fuente: elaboración propia 
La técnica utilizada para la toma de muestras fue locativa, es decir, se toma una parte del suelo con riesgo de deslizamiento y progresivamente, con intervalos de tiempo, se le agrega una cantidad de mililitros de agua para ver su reacción. Luego de la unión de todas estas herramientas, se llega a los resultados obtenidos que se podrán ver en el siguiente apartado.

\section{Resultados}

Se tomaron 35 muestras de la estación base desde las 05:40 p.m. hasta las 07:22 p.m. del 21 de enero del 2018, con un espacio de tres minutos entre cada muestra, en 900 gramos de suelo de la localidad. Se compararon cuatro variables: tiempo, temperatura, humedad y mililitros de agua. En la figura 7, evidenciamos el archivo que arrojó el montaje, con nombre datos.txt, generado en un bloc de notas.

Para este ejemplo, se toman las muestras que generan alerta según la escala determinada antes.

Después de la generación del archivo, se extrae la tarjeta micro SD en la que se creó el archivo datos. txt y se pasa al respectivo análisis, construyendo gráficas de comparación entre las variables mencionadas antes. A continuación, se observan las gráficas de temperatura, humedad y humedad versus mililitros de agua, que determinan cuándo se generó la alerta y cuáles fueron los parámetros.

Enero 21 del 2018 05:40 pm,Temperatura=15,Humedad=1 con $50 \mathrm{ml}$ de agua Enero 21 del 2018 05:43 pm,Temperatura=15,Humedad=10 con $50 \mathrm{ml}$ de agua Enero 21 del 2018 05:46 pm,Temperatura=15,Humedad=46 con $100 \mathrm{ml}$ de agua Enero 21 del 2018 05:58 pm,Temperatura=13,Humedad=66 con $150 \mathrm{ml}$ de agua Enero 21 del 2018 06:01 pm,Temperatura=13,Humedad=74 con $150 \mathrm{ml}$ de agua Enero 21 del 2018 06:40 pm,Temperatura=13,Humedad=84 con $150 \mathrm{ml}$ de agua Enero 21 del 2018 06:43 pm,Temperatura=13,Humedad=83 con $200 \mathrm{ml}$ de agua Enero 21 del 2018 06:46 pm,Temperatura=13,Humedad=82 con $200 \mathrm{ml}$ de agua Enero 21 del 2018 06:49 pm,Temperatura=13,Humedad=82 con $200 \mathrm{ml}$ de agua Enero 21 del 2018 06:52 pm,Temperatura=13,Humedad=89 con $200 \mathrm{ml}$ de agua Enero 21 del 2018 06:55 pm,Temperatura=13,Humedad=75 con $200 \mathrm{ml}$ de agua Enero 21 del 2018 06:58 pm,Temperatura=13,Humedad=74 con $200 \mathrm{ml}$ de agua

Figura 7. Muestras tomadas por la estación base sistema de alerta temprana por movimiento en masa inducido por lluvia para la localidad de Ciudad Bolívar

Fuente: elaboración propia

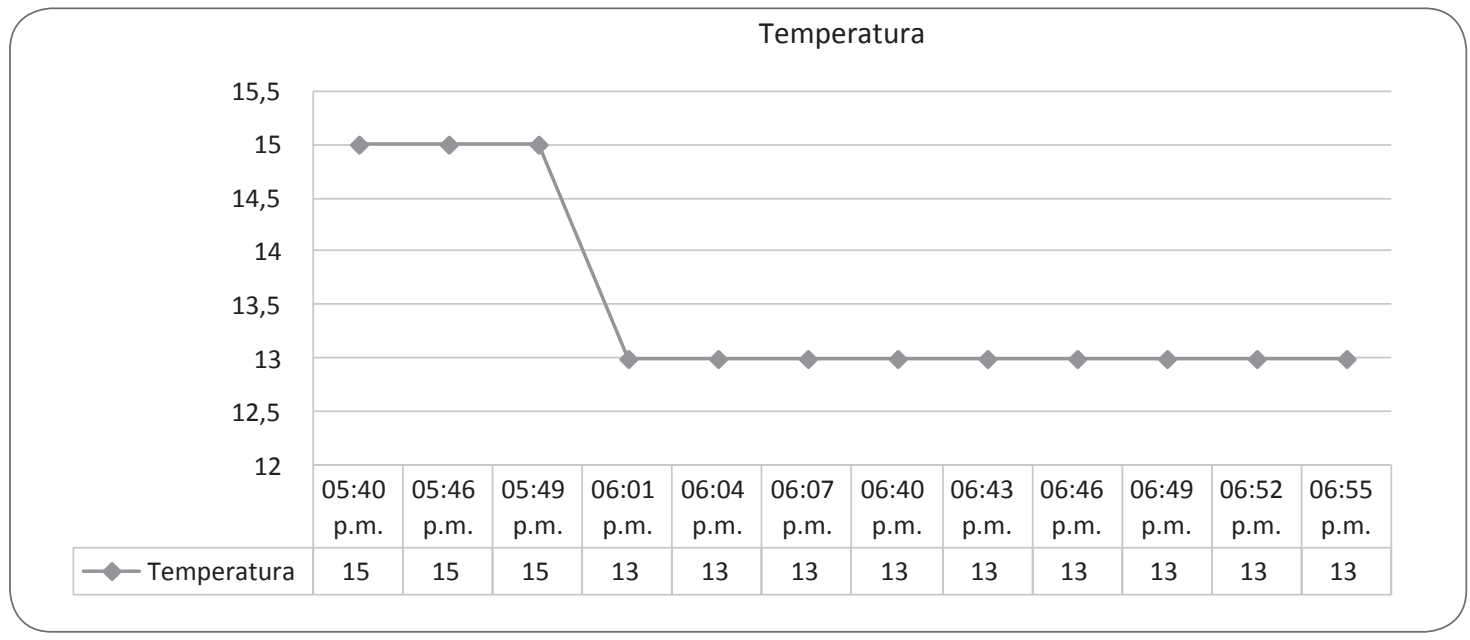

Figura 8. Gráfica de temperatura versus tiempo

Fuente: elaboración propia 
En la figura 9, se evidencia la humedad generada después de verter $200 \mathrm{ml}$ de agua en los 900 gramos de tierra en diferentes horas. Logramos ver cómo aumenta la humedad generando alerta de 6:40 p.m. a 6:52 p.m. de posibles deslizamientos según nuestra escala.
En la figura 10, encontramos la relación entre humedad y mililitros de agua, que en total fueron $200 \mathrm{ml}$, y a las 6:40 p.m. sobrepasa las mediciones de alerta generada.

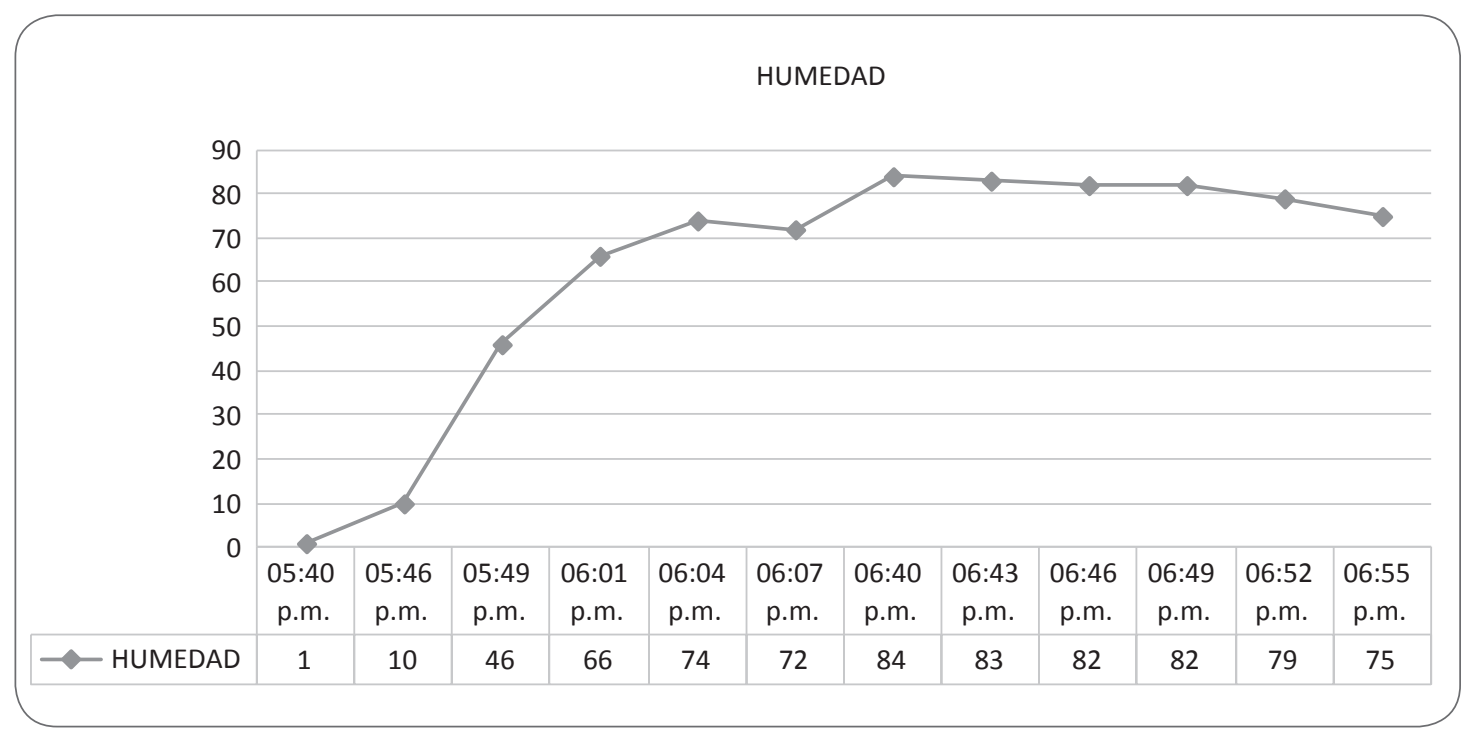

Figura 9. Gráfica de humedad versus tiempo tomada por la estación base Fuente: elaboración propia

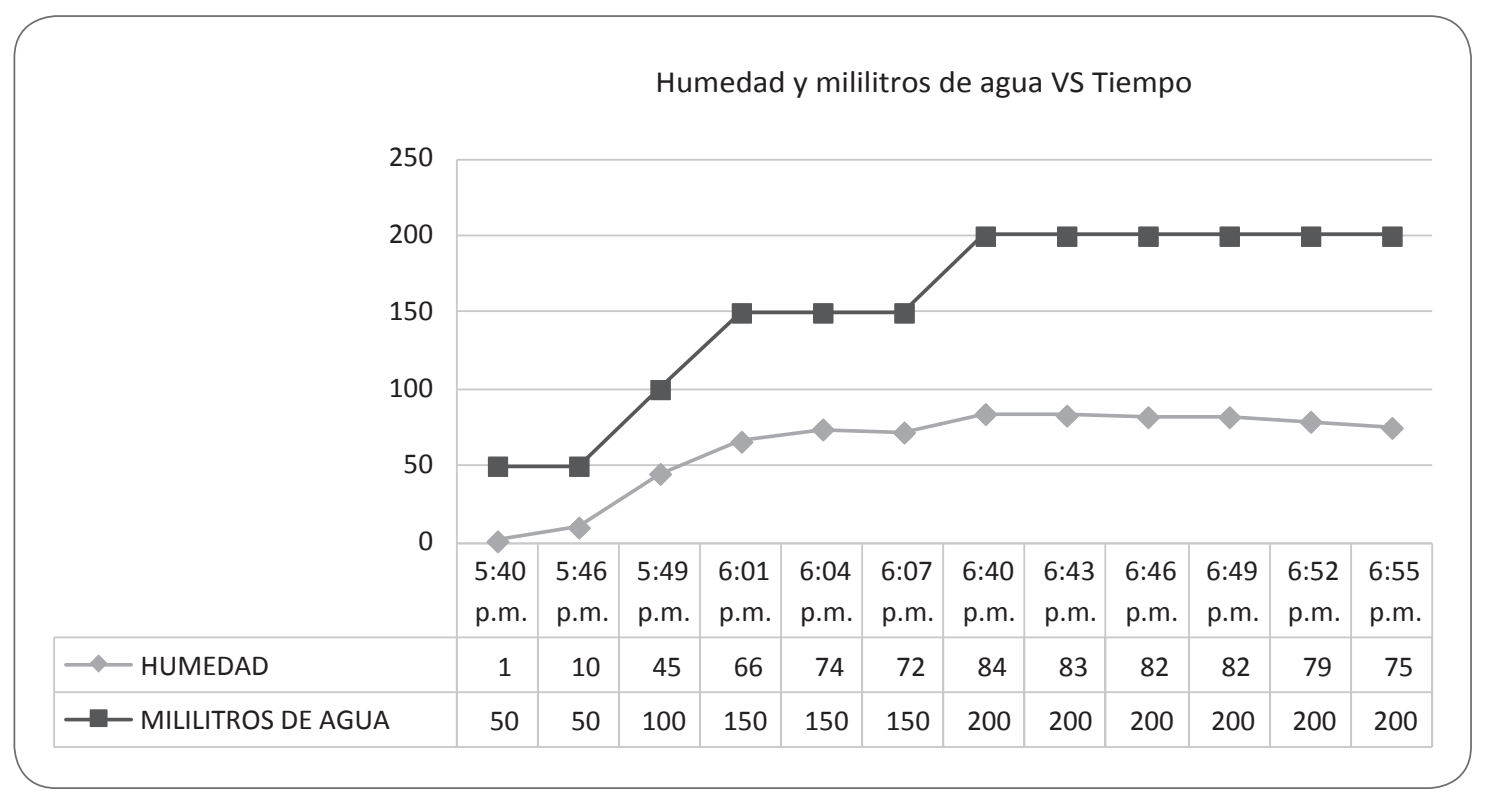

Figura 10. Gráfica de humedad versus mililitros de agua Fuente: elaboración propia 


\section{Discusiones}

Las discusiones que se generan alrededor de este tema fue necesario abordarlas desde la raíz. Cuando se realiza el Plan de Ordenamiento Territorial en Bogotá, se delimitan los lugares en donde se puede construir más de un piso o los lugares donde esta rotundamente prohibido construir, ya sea porque es una reserva natural, un humedal o representa un peligro inminente para las personas.

Se sabe que en los lugares altos de la ciudad, es decir, hacia los cerros, están ubicados estos lugares peligrosos con prohibición del Distrito para construir. Pero aún hay personas, en su mayoría desplazadas, que construyen artesanalmente, generando riesgo de deslizamiento inducido por lluvia que pueden perjudicarlos.

La discusión consiste en qué debe hacer el gobierno para evitar la construcción de este tipo de viviendas en zonas de riesgo. Aparte de la solución brindada en este artículo de un sistema de alerta temprana por deslizamiento de tierra inducido por lluvia, es necesario otra solución alterna que brinde mayor seguridad y reduzca el número de muertes anuales por deslizamiento de tierra.

\section{Conclusiones}

- El sistema de alerta temprana por movimiento en masa inducido por lluvia para la localidad de Ciudad Bolívar, como red de sensores guiada por una estación base, muestra las alertas generadas por medio de gráficas, dando tiempo suficiente para informar a las personas que se encuentren en el sector afectado y evitar muertes/damnificaciones por deslizamiento de tierra.

- Trabajar con Arduino brinda una gran ayuda y facilidad al momento de programar para las personas que no están tan informadas del tema o que simplemente no tiene ningún conocimiento en programación; además de brindar una interfaz gráfica fácil de utilizar en cualquier idioma.

- La tecnología es de vital importancia para mejorar la calidad de vida del ser humano. Después de la investigación y elaboración del artículo, se concluye que la mejor herramienta tecnológica para la recaudación de datos, en este tipo de ámbito, son los sensores y los nodos. Esto es porque brindan una mayor estabilidad y un menor riesgo de que se pierda la información; es claro que para que funcione perfectamente se requiere de un conjunto de factores.
- El principal aporte de este artículo consiste en que muestra una forma de alertar a la comunidad en caso de probabilidad de deslizamiento, por medio de la tecnología que hoy en día está a mano de cualquier persona; además de informar, prevenir y alertar, es abrir la puerta a que este tipo de ayudas llegue a los sectores más deprimidos de la ciudad, donde la violencia y la pobreza es el diario vivir.

- Otros de los aportes del proyecto es el bajo costo de fabricación de dispositivos como la estación base, ya que no se requiere de gran infraestructura, maquinaria o mucho dinero para implementarlo en la comunidad, y sí salvaría muchas vidas en estos lugares de alto riesgo. Lo que se quiere con este artículo, además de mostrar una opción de tecnología para el bien común, es decirles a las personas el riesgo que conlleva construir en lugares prohibidos.

\section{Referencias}

[1] L. Bravo, R. J. Hernández, I. Llatas y A. Salcedo, "Desarrollo de un sistema de alerta temprano comunitario en el estado Vargas". Temas de Coyuntura, no. 61, pp. 169-178, julio 2010 [En línea]. Disponible en: http://revistasenlinea.saber.ucab.edu.ve/ temas/index.php/temasdecoyuntura/article/download/1928/1667.

[2] C. A. Gálvez-Hooper, "Evaluación del riesgo a inundaciones y deslizamientos en la parte alta de la cuenca del río Chiriquí Viejo, Panamá”, Tesis de maestría, Centro Agronómico Tropical de Investigación y Enseñanza, Turrialba, Costa Rica, 2013 [En línea]. Disponible en: http://orton.catie.ac.cr/repdoc/A5963E/ A5963E.PDF

[3] D. Yábar, "Metodología para la planificación de un Sistema de Alerta Temprana (SAT) a inundaciones para la región de Madre de Dios, Perú", Tesis de maestría, Centro Agronómico Tropical de Investigación y Enseñanza, Turrialba, Costa Rica, 2014 [En línea]. Disponible en: http://repositorio.bibliotecaorton.catie.ac.cr/bitstream/handle/11554/7129/ Metodologia_para_la_planificacion_de_un_sistema.pdf? sequence $=1$ \&isAllowed $=y$

[4] C. Castro, C. Marquardt y Á. Zúñiga, "Peligros naturales en geo sitios de interés patrimonial en la costa sur de Atacama”. Rev. Geogr. Norte Gd., no. 45, pp. 21-39, mayo 2010 [En línea]. doi: http://dx.doi.org/ $10.4067 /$ S0718-34022010000100002 
[5] C. A. Peña-Rincón, "Monitoreo y Modelo Sistémico a Deslizamiento Superficial de Suelos para Manizales", Tesis doctoral, Universidad Nacional de Colombia, Caldas, Colombia, 2014 [En línea]. Disponible en: http://www.bdigital.unal.edu. co/48755/1/3166172.2014.pdf

[6] M. A. Pradenas, "Umbral empírico de deslizamiento por precipitación para la provincia de Concepción", Trabajo de grado, Universidad del Bio-Bio, Concepción, Chile, 2014 [En línea]. doi: http://dx.doi. org/10.4067/S0718-34022010000100002

[7] C. Cardozo, "Zonación de susceptibilidad por procesos de remoción en masa en la cuenca del río Tartagal, Salta (Argentina)", Tesis de Maestría, Universidad Nacional de Córdoba, Córdoba, Argentina, 2013 [En línea]. Disponible en: http://ig.conae.unc. edu.ar/wp-content/uploads/sites/68/2017/08/2010_ Cardozo-Paola.pdf

[8] C. F. Proaño-Pachucho, "Análisis de los componentes estructurales y funcionales para el diseño de un sistema de alerta integral a nivel institucional, por potenciales inundaciones en el Distrito Metropolitano de Quito", Tesis de maestría, Universidad Andina Simón Bolívar, Quito, Ecuador, 2016 [En línea]. Disponible en: http://hdl.handle.net/10644/5405
[9] L. Carbajal, "Riesgo de deslizamientos ocasionados por las precipitaciones en la micro cuenca de comas - concepción - Junín" Universidad nacional del centro del Perú facultad de ciencias forestales y del ambiente, PP. 15 a 83, Perú, 2012 [En línea]. Disponible en: http://repositorio.uncp.edu.pe/handle/ $\mathrm{UNCP} / 2615$

[10] A. Albuja y M. Ceballos, "Desplazamiento urbano y migración en Colombia". Revista Migraciones Forzadas, no. 34, pp. 10-11, marzo 2010 [En línea]. Disponible en: https://rua.ua.es/dspace/bitstream/10045/13771/1/RMF_34_04.pdf

[11] Secretaría Distrital de Planeación, Diagnóstico de los aspectos físicos, demográficos y socioeconómicos. Bogotá: Secretaría Distrital de Planeación, 2009.

[12] N. García, "Mapa de amenaza por riesgo de deslizamiento". Tesis de grado, Pontificia Universidad Javeriana, Bogotá, Colombia, 2013 [En línea]. Disponible en: https://repository.javeriana.edu.co/ bitstream/handle/10554/12661/GarciaRamosNicoleAndrea2013.pdf? sequence $=1$ \&isAllowed $=y$

[13] M. Hermelin, Entorno natural de 17 ciudades de Colombia. Medellín: Fondo Editorial Universidad Eafit, 2007. 